\title{
Reviewer Recognition and Acknowledgement
}

The International Journal for Students as Partners' Editorial Board extends its thanks to the community of reviewers that have contributed to past and current editions of the journal.

Sophia Abbot
Anita Acai
Ashley Akenson
Melanie Bahti
Eric Balaam
Susanna Barrineau
Amani Bell
Nina Bernius
Sonal Bhalla
Dan Bishop
Julie Blackwell Young
Stephen Bloch-Schulman
Alyson Brown
Duygu Candarli
Alice Cassidy
Ming Cheng
Barbara Colledge
Leanne Coombe
Duncan Cross
Roisin Curran
Sinead D'Silva
Shane Dawson
Mollie Dollinger
Michael Draper
Lucie Dvorakova
Alexander Dwyer
Peter Felten
Nancy Fenton
Abbi Flint
Susan He
Paul Hernandez-Martinez
Elizabeth Ho
Huang Hoon Chng
Julia Hope

Sophia Abbot

Anita Acai

Ashley Akenson

Melanie Baht

Susanna Barrineau

mani Bell

Nina Bernius

Sonal Bhalla

Julie Blackwell Young

Alyson Brown

Duygu Candarli

Alice Cassidy

Ming Cheng

Barbara Colledge

Duncan Cross

Roisin Curran

Shane Dawson

Michael Draper

Michael Drape

Lucie Dvorakova

Peter Felten

Nancy Fenton

Susan He

Paul Hernandez-Martinez

Huang Hoon Chng

Julia Hope

\author{
Rebecca Khanna \\ Katie Kinsella \\ Sabrina Kirby \\ Alyona Koulanova \\ Ketevan Kupatadze \\ Rebecca LeBard \\ Tanya Lubicz-Nawrocka \\ Margy MacMillan \\ Jenny Marie \\ Clarke Mathany \\ Susannah McGowan \\ Victoria McKinnon \\ Luke Millard \\ Aisha Mohamed \\ Liz Mossop \\ Damon Motz-Storey \\ Simon Pratt-Adams \\ Sarah Preedy \\ Jane Pritchard \\ Sarah Rasmussen \\ Naima Raza \\ Jessica Riddell \\ Stuart Russell \\ Jennifer Schafer \\ Hilary Schuldt \\ Christine Slade \\ Jo Smedley \\ Karen Smith \\ Rachel Spronken-Smith \\ Raksha Sule \\ Stephanie Verkoeyen \\ Phil Wilkinson \\ Cassia Wojcik \\ Donna Ziegenfuss
}

